\title{
Downregulation of active caspase 8 as a mechanism of acquired TRAIL resistance in mismatch repair-proficient colon carcinoma cell lines
}

\author{
CAROLINE M.M. VAN GEELEN, BODVAEL PENNARUN, WYTSKE BOERMA-VAN EK, PHUONG T.K. LE, \\ DIANA C.J. SPIERINGS, ELISABETH G.E. DE VRIES and STEVEN DE JONG
}

\author{
Department of Medical Oncology, University Medical Center Groningen, \\ Hanzeplein 1,9713 GZ, Groningen, The Netherlands
}

Received May 12, 2010; Accepted June 29, 2010

DOI: 10.3892/ijo_00000755

\begin{abstract}
Tumor necrosis factor-related apoptosis-inducing ligand (TRAIL) triggers the apoptotic cascade in various colon cancer cell lines after binding to the membrane receptors DR4 and DR5. However, not all cancer cell lines are sensitive to the therapeutic recombinant human TRAIL (rhTRAIL). To investigate the causes of TRAIL resistance in colon cancer cell lines, models have been developed, mostly in mismatch repair-deficient cells. These cells are prone to mutations in genes containing tandem repeat, including pro-apoptotic protein Bax. We therefore investigated the mechanism underlying TRAIL resistance acquisition in a mismatch repairproficient colon carcinoma cell line. The TRAIL-resistant cell line SW948-TR was established from the TRAIL-sensitive cell line SW948 by continuous exposure to rhTRAIL, and exhibited 140-fold less sensitivity to rhTRAIL in a cell viability assay. Resistance was stable for over a year in the absence of rhTRAIL. Both cell lines had similar TRAIL receptor cell membrane expression levels. Treatment with the protein synthesis inhibitor cycloheximide sensitized SW948-TR to rhTRAIL-induced apoptosis, indicating that the functionality of the TRAIL receptors was maintained. In SW948-TR, procaspase 8 protein levels but not mRNA levels were notably lower than in SW948. Downregulation of c-FLIP with short interfering RNA (siRNA) sensitized SW948-TR cells to rhTRAIL while caspase 8 siRNA decreased rhTRAIL sensitivity in SW948, indicating the importance of the caspase 8/c-FLIP ratio. Proteasome inhibition with MG132 did not restore basic procaspase 8 levels but stabilized cleaved caspase 8 in rhTRAIL-treated SW948-TR cells. Altogether, our results suggest that colon cancer cells can acquire rhTRAIL resistance by primarily reducing the basal procaspase $8 / \mathrm{c}$ FLIP ratio and by increasing active caspase 8 degradation
\end{abstract}

Correspondence to: Dr S. De Jong, Department of Medical Oncology, University Medical Center Groningen, Hanzeplein 1, 9713 GZ Groningen, The Netherlands

E-mail: s.de.jong@int.umcg.nl

Key words: TRAIL, DR4, DR5, caspase 8, FLIP, drug resistance, apoptosis after rhTRAIL treatment. Proteasome inhibitors can effectively overcome acquired rhTRAIL resistance in mismatch repairproficient colon cancer cells.

\section{Introduction}

TRAIL (tumor necrosis factor-related apoptosis-inducing ligand) is a member of the TNF family with interesting anticancer activity $(1,2)$. Binding of TRAIL to its two apoptosisinducing receptors DR4 and DR5 induces formation of a complex called the death-inducing signaling complex (DISC), leading to caspase-dependent stimulation of the extrinsic pathway of apoptosis. The therapeutic agent recombinant human (rh)TRAIL has been shown to induce apoptosis in many types of human cancer cell lines in vitro, and also prevents tumor growth in preclinical models in vivo. By activating both the extrinsic and the intrinsic pathway of apoptosis, combination treatment of rhTRAIL and chemotherapy can also induce apoptosis in drug-resistant cell lines both in vitro and in vivo in human tumor xenograft mouse models. A phase I study has consequently been initiated with rhTRAIL and demonstrated safety of this agent (3-5). In a phase II study in heavily pre-treated colon cancer patients, the best response to an anti-DR4 antibody was stable disease in $32 \%$ cases $(6)$.

About $60 \%$ of cancer cell lines are moderately to completely resistant to rhTRAIL in vitro (7). Understanding the molecular basis of cancer cell resistance to TRAIL-induced apoptosis may hold the key to optimal TRAIL receptortargeted combination therapies and the development of novel treatment initiatives. Several key components for TRAIL sensitivity have been identified. This includes downstream pro- and anti-apoptotic proteins of the Bcl-2 family such as Bax, Bak and Bcl-2, proteins inhibiting efficient DISC formation such as c-FLIP and finally caspase inhibitors such as X-linked inhibitor of apoptosis protein (XIAP) (8). In DNA mismatch repair (MMR)-deficient tumor colon cancer cell line, the acquisition of TRAIL resistance after extended exposure to the ligand was linked to the accumulation of Bax mutations resulting in $\mathrm{Bax}^{-/-}$phenotype, both in vitro and in vivo (9). MMR deficiency leads to microsatellite instability (MSI). MSI-positive tumors have a high rate of frame-shift mutations in a number of genes containing tandem repeat sequences, 
including the pro-apoptotic gene Bax $(10,11)$. Apart from hereditary non-polyposis colon cancer tumors, which accounts for approximately $2-5 \%$ of colorectal cancer cases, MSI as a result of MMR deficiency is present in around $15 \%$ of sporadic colon tumors (12). Because the majority of the colon cancers are MMR-proficient, this group of tumors deserves attention as well. By retroviral transfection of a human cDNA library in the TRAIL-sensitive colon cancer cells line SW480, others previously reported that $\mathrm{FLIP}_{\mathrm{s}}$ and $\mathrm{Bcl}-\mathrm{X}_{\mathrm{L}}$ overexpression could induce TRAIL resistance (13). To further identify the components for rhTRAIL sensitivity in MMR-proficient colon carcinoma cells, we established a rhTRAIL resistant sub-line by prolonged exposure of the previously characterized TRAILsensitive SW948 colon carcinoma cell line $(14,15)$. The mechanisms of TRAIL resistance were analyzed in this distinct isogenic background.

\section{Materials and methods}

Reagents. RPMI-1640 medium was obtained from Life Technologies (Breda, The Netherlands) and fetal calf serum (FCS) from Bodinco BV (Alkmaar, The Netherlands). 3 (4,5-dimethyl-thiazol-2-yl) 2,5-diphenyltetrazolium bromide (MTT)-solution, cycloheximide was purchased from SigmaAldrich Chemie BV (Zwijndrecht, The Netherlands). RhTRAIL was produced non-commercially in cooperation with IQ-Corp. (Groningen, The Netherlands) following a protocol described earlier (7). The TRAIL-receptor antibodies used for flow cytometry were obtained from Immunex (Seattle, WA, USA). Caspase 9 inhibitor zLEHDfmk, caspase 8 inhibitor zIETD-fmk, broad-spectrum caspase inhibitor zVAD-fmk and proteasome inhibitor MG132 were obtained from Calbiochem (Breda, The Netherlands). Oligofectamine reagent was purchased from Invitrogen BV (Breda, The Netherlands). The NFKB inhibitor SN-50 was obtained from Biomol (Tebu-bio, Heerhugowaard, The Netherlands).

Cell lines. The TRAIL-sensitive colon carcinoma SW948 cell line was cultured in Leibovitz L15-RPMI-1640 (1:1) enriched with $10 \% \mathrm{FCS}, 0.05 \mathrm{M}$ pyruvate, $0.1 \mathrm{M}$ glutamine and $0.025 \%$ B-mercaptoethanol at $37^{\circ} \mathrm{C}$ in a humidified atmosphere with $5 \% \mathrm{CO}_{2}$. SW948 was harvested by treatment with protease XXIV for $5-10 \mathrm{~min}$ at $37^{\circ} \mathrm{C}$. In order to obtain a TRAIL-resistant sub-line, SW948 was exposed continuously to $1.0 \mu \mathrm{g} / \mathrm{ml} \mathrm{rhTRAIL}$ and incubated at $37^{\circ} \mathrm{C}$ to allow resistant cells to grow. After 14 days these cells were cultured in the presence of $2.5 \mu \mathrm{g} / \mathrm{ml}$ rhTRAIL twice a week. After 3 months the stable TRAIL-resistant cell line SW948-TR was established and cultured similarly to its parental cell line. In the absence of rhTRAIL in the culture media SW948-TR remains resistant to rhTRAIL for at least 75 passages. Based on cell viability assay, this cell line is at least 140 -fold more resistant to rhTRAIL than the sensitive parental cell line.

Sub-cloning of SW948-TR. SW948-TR was harvested by treatment with protease XXIV for $5-10 \mathrm{~min}$ at $37^{\circ} \mathrm{C}$, washed once in Leibovitz L15-RPMI-1640 (1:1) without FCS. A single cell suspension was obtained by resuspending the cells repeatedly through an 18-gauge needle.
Cells were diluted in 2 vol HAM-DME (1:1) with $20 \%$ FCS and 1 vol conditioned Leibovitz L15-RPMI-1640 (1:1) medium with $10 \%$ FCS. The cell suspension was kept at $37^{\circ} \mathrm{C}$, then $1 / 10 \mathrm{vol}$ of pre-warmed agarose (final concentration $0.3 \%$ ) was added and carefully mixed. Cell suspension $(2 \mathrm{ml})$ was added to each Petri dish (200 or 20 cells) which were kept at $4^{\circ} \mathrm{C}$ for $1 \mathrm{~h}$. Then, Petri dishes were placed at $37^{\circ} \mathrm{C}$ in a humidified atmosphere with $5 \% \mathrm{CO}_{2}$. After 14 days individual visible clones were transferred to a 24 -well plates and cultured in Leibovitz L15-RPMI-1640 (1:1) medium with $10 \%$ FCS. Growing clones were harvested by treatment with protease XXIV for $5-10 \mathrm{~min}$ at $37^{\circ} \mathrm{C}$ and cultured as described for SW948-TR.

An alternative sub-cloning approach was minimal dilution of SW948-TR in 2 vol Leibovitz L15-RPMI-1640 (1:1) medium with $10 \%$ FCS and 1 vol conditioned Leibovitz L15-RPMI-1640 (1:1) medium with 10\% FCS. Cell suspension was diluted and thereafter $100 \mu 1$ was added to each well of a 96-well plates. After 14 days, cells from wells with single colony were harvested with protease XXIV for 5-10 min at $37^{\circ} \mathrm{C}$, transferred to a well of a 24 -well plates, and incubated as described above.

Cytotoxicity assay. The microculture tetrazolium assay was used to determine cytotoxicity. SW948 and SW948-TR cells were incubated in a total volume of $200 \mu$ l. Treatment consisted of continuous incubation with various rhTRAIL concentrations. After an incubation period of $96 \mathrm{~h}, 20 \mu \mathrm{l}$ of MTT-solution [5 mg/ml phosphate-buffered saline (PBS): $6.4 \mathrm{mM} \mathrm{Na}_{2} \mathrm{HPO}_{4}$; $1.5 \mathrm{mM} \mathrm{KH}{ }_{2} \mathrm{PO}_{4} ; 0.14 \mathrm{mM} \mathrm{NaCl} ; 2.7 \mathrm{mM} \mathrm{KCl} ; \mathrm{pH}$ 7.2] was added for $3.75 \mathrm{~h}$. Subsequently, plates were centrifuged and the supernatant aspirated. After dissolving the formazan crystals by adding dimethyl sulfoxide (Merck, Amsterdam, The Netherlands), plates were read immediately at $520 \mathrm{~nm}$ using a microtiter well spectrometer (Bio-Rad microplate reader, Bio-Rad Laboratories BV, Veenendaal, The Netherlands). Controls consisted of media without cells. Cell survival was defined as the growth of treated cells compared to untreated cells. The $\mathrm{IC}_{50}$ was defined as the drug concentration inhibiting survival by $50 \%$. Mean cytotoxicity was calculated from three independent experiments each performed in quadruplicate.

SDS-polyacrylamide gel electrophoresis and Western blotting. Preparation of protein lysates and Western blot analysis was performed as described previously (15). To detect poly-ADP-ribose-polymerase (PARP), FADD, X-linked inhibitor of apoptosis protein (XIAP), FLICE-like inhibitory protein (FLIP), caspases 3, 9, 8 and 10, DR4, DR5, Bid, Bax, Bak, Bcl- $\mathrm{X}_{\mathrm{L}}, \mathrm{Bcl}-2$, Bid and actin the following antibodies were used: rabbit anti-PARP from Roche applied science (Mannheim, Germany), rabbit mouse-anti-FADD and mouse anti-XIAP from Transduction Laboratories (Lexington, KY), rabbit anti-caspase 3, rabbit anti-Bid and rabbit anti-caspase 9 from Pharmingen (Becton-Dickinson, Erebodegem-Aalst, Belgium), goat anti-DR4, mouse anti-Bcl-2, rabbit anti$\mathrm{BclX}_{\mathrm{L}}$, rabbit anti-Bax (N20), goat anti-Bak (N20) from Santa Cruz Biotechnology (Santa Cruz, CA, USA) rabbit anti-DR5 and rabbit anti-survivin from Oncogene Research Products (Calbiochem-Novabiochem, Germany). Mouse 
anti-caspase 8 was purchased from Cell Signaling Technology (Leusden, the Netherlands). Mouse anti-caspase 10 , rabbit anti-cIAP-1 and rabbit anti-cIAP-2 were obtained from R\&D Systems (Abingdon, UK). Mouse anti-FLIP NF6 was kindly provided by Dr M. Peter (Chicago, IL, USA). Mouse anti-actin was obtained from ICN Biomedicals (Zoetermeer, The Netherlands). The secondary antibodies were labeled with horseradish peroxidase (all from Dako, Glostrup, Denmark) and chemiluminesence was detected using the ECL-chemiluminescence kit or with the LumiLight Plus Western blotting kit (Roche Diagnostics, Mannheim, Germany). Western blot analyses were performed at least three times. Protein concentrations were determined with the Bradford assay. In all experiments samples containing 15 or $20 \mu \mathrm{g}$ lysate were used, and membranes were stained with Ponceau $\mathrm{S}$ to check for equal protein loading.

Flow cytometry. Analysis of TRAIL-receptor membrane expression was performed with a flow cytometer (Epics Elite, Coulter-Electronics, Hialeah, FL, USA) and cells were stained as described earlier (15). The following antibodies were used: for DR4, huTRAILR1-M271, for DR5, huTRAILR2-M413, for DcR1, huTRAILR3-M430, and for DcR2, huTRAILR4-M444. Membrane receptor expression is shown as mean fluorescent intensity (MFI) of all analyzed cells. Membrane expression was observed as an increase in fluorescence intensity for the whole analyzed cell population. All experiments were performed at least three times.

Apoptosis assay. For each cell line 10,000 cells were seeded in 96 -well plates. Apoptosis was identified by staining nuclear chromatin with acridine orange (AO), identifying morphological changes by fluorescence microscopy. Apoptosis was expressed as percentage apoptotic cells in a culture. Apoptosis experiments were performed at least three times.

RNA interference. Small interfering RNAs (siRNAs) specific for human c-FLIP were designed conforming to the sequence $\mathrm{AA}(\mathrm{N} 19) \mathrm{TT}$, where AA and TT are present in the c-FLIP open reading frame at a spacing of 19 nucleotides. Doublestranded RNA molecules specific for c-FLIP (sense: 5'-GAG GUA AGC UGU CUG UCG GdTdT-3', anti-sense: 5'-CCG ACA GAC AGC UUA CCU CdTdT-3') or caspase 8 (sense: 5'-CUA CCA GAA AGG UAU ACC UdTdT-3', anti-sense: 5'-AGG UAU ACC UUU CUG GUA GdTdT-3') were synthesized by Eurogentec (Seraing, Belgium). Single stranded RNA molecules specific for the luciferase (Luc) gene were used as control (16). The sequences for Luc RNA molecules were 5'-CUU ACG CUG AGU ACU UCG AdTdT-3' (sense) and 5'-UCG AAG UAC UCA GCG UAA GdTdT-3' (antisense). To form RNA duplexes, $20 \mu \mathrm{M}$ of both single-stranded RNAs were incubated in annealing buffer supplied by Eurogentec (50 mM Tris, pH 7.5-8.0, $100 \mathrm{mM} \mathrm{NaCl}$ in RNasefree distilled water) for $1-5 \mathrm{~min}$ at $90^{\circ} \mathrm{C}$ and cooled down to room temperature in $\sim 1 \mathrm{~h}$. Cells were harvested with protease and transfected in 6 -well plates $\left(0.3 \times 10^{6}\right.$ cells/well $)$ with 2.5-10 $\mu \mathrm{l}$ of $20 \mu \mathrm{M}$ siRNA duplexes using oligofectamine reagent according to the manufacturer's instructions. The next day, cells were harvested and seeded in 96- or 6-well plates for apoptosis assay or protein isolation respectively. After $48 \mathrm{~h}$ siRNA transfected cells were incubated with $0.1 \mu \mathrm{g} / \mathrm{ml} \mathrm{rhTRAIL}$ for $5 \mathrm{~h}$. After treatment, the percentage apoptosis was determined by AO apoptosis assay or the cells were lysed for protein analysis.

Real-time PCR. Total RNA was isolated by guanidine isothiocyanate-phenol-chloroform extraction using TRIzol (Invitrogen) according to the manufacturer's protocol. Total RNA was purified with the RNeasy mini kit (Qiagen, Leusden, The Netherlands) according to the manufacturer's instructions. Trace amounts of DNA contamination were removed by on-column DNase I digestion following the manufacturer's recommendations.

Yield and quality of the total purified RNA was assessed by measuring A260/280 $\mathrm{nm}$ and 260/230 nm ratio on a nanodrop ND-1000 spectrophotometer (Nanodrop, Isogen Life Science B.V., IJsselstein, The Netherlands) and by RNA gel electrophoresis visualization of $18 \mathrm{~S}$ and $28 \mathrm{~S}$ rRNA bands. cDNA was synthesized from $800 \mathrm{ng}$ purified RNA as described by the manufacturer's protocol (Life Technologies) using oligo $(\mathrm{dT})_{11}$ primers and MMLV transcriptase. Prior to the RT-PCR, purity and integrity of the synthesized cDNA was examined by qualitative RT-PCR for the housekeeping reference gene coding for the enzyme glyceraldehyde-3phosphate dehydrogenase (GAPDH). Real-time RT-PCR was performed in 96-well plates using the SYBR Green method on a MyiQ real-time detection system (all from Bio-Rad) using GAPDH as an internal control. A gradient RT-PCR was performed to assess primer specificity and to optimize annealing temperature $\left(T_{a n n}\right)$ for each set of gene specific primers. The sequences for the primers used in real-time PCR of caspase 8 were GGAGCTGCTCTTCCGAATTA (forward) and GCAGGTTCATGTCATCATCC (reverse), for FLIP and those of GAPDH were CACCACCATGGA GAAGGCTGG (forward) and CCAAAGTTGTCATGG ATGACC (reverse). Amplification of the samples was carried out in triplicate in a final reaction volume of $25 \mu 1$, containing 12.5 $\mu$ I IQ SYBR Green Supermix (BioRad), $1 \mu 1$ of each gene specific primer $(5 \mu \mathrm{M})$ and $5 \mu 1 \mathrm{cDNA}$ (1:50). The thermocycling program used for each real time RT-PCR consisted of an initial $3 \mathrm{~min}$ denaturation at $95^{\circ} \mathrm{C}$, followed by 40 cycles of $15 \mathrm{sec}$ denaturation at $95^{\circ} \mathrm{C}, 20 \mathrm{sec}$ primer annealing at the primer specific Tann and $30 \mathrm{sec}$ fragment elongation at $72^{\circ} \mathrm{C}$. Fluorescence data was acquired during the fragment elongation step at $72^{\circ} \mathrm{C}$. Presence of unique reaction products was determined from the melting curves obtained at the end of 40 cycles of amplification.

To determine RT-PCR efficiency and initial starting quantity of the samples, a standard curve was generated using a 1:3 serial dilution from total starting cDNA sample. Water controls were included to check for DNA contamination. Differences in the amount of starting cDNA between samples were corrected using GAPDH as a housekeeping reference gene.

Statistical analysis. Statistical analysis was performed using the Student's t-test. P-values $\leq 0.05$ were considered to be significant. 
A
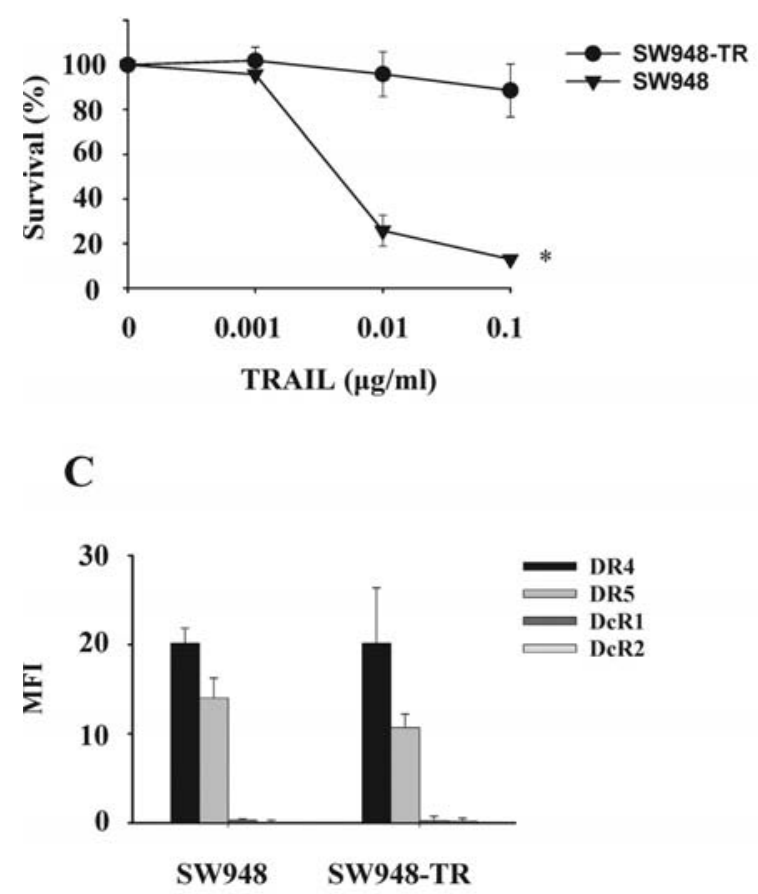

B
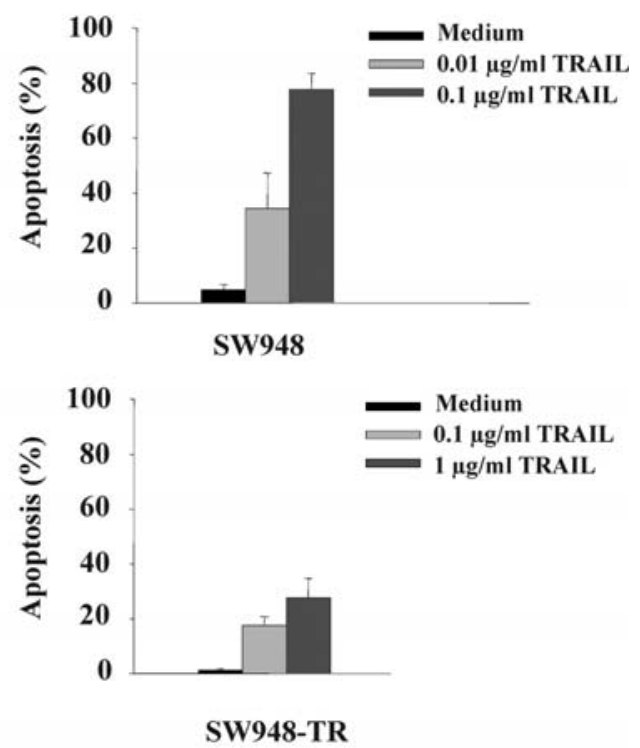

D

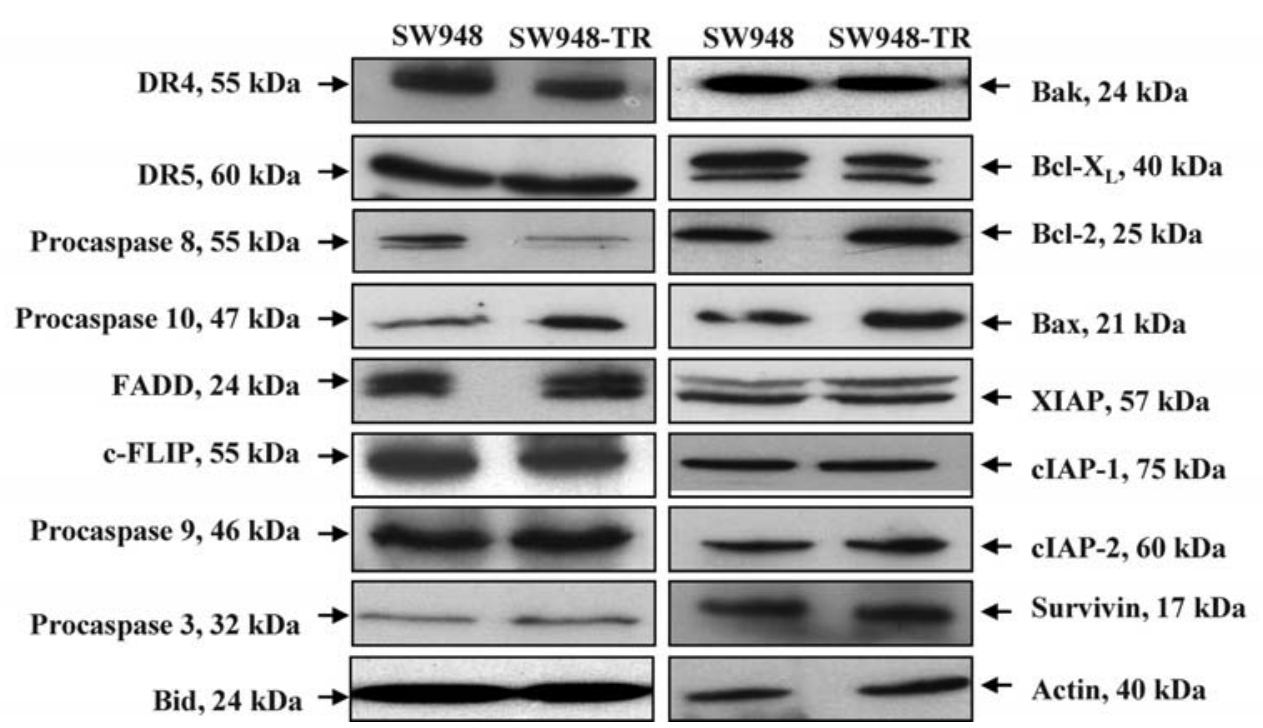

Figure 1. The SW948-TR cell line is less sensitive to rhTRAIL than its parental SW948 cell line and expressed lower procaspase 8 protein levels. (A) Survival (\%) of SW948 and SW948-TR, after continuous incubation with rhTRAIL as measured by cytotoxicity assays. Values are mean \pm SD of at least three independent experiments. "SW948 differs from SW948-TR at 0.01-1 $\mu \mathrm{g} / \mathrm{ml}$ rhTRAIL ( $\mathrm{p} \leq 0.05$ ). (B) Apoptosis (\%) of SW948 (top) and SW948-TR (bottom), after incubation with various concentrations of rhTRAIL for $5 \mathrm{~h}$, as assessed following AO staining. Values are mean \pm SD of at least three independent experiments. (C) Basic membrane expression of the TRAIL receptors DR4 (black bars), DR5 (dark gray bars), DcR1 (white bars), and DcR2 (light gray bars) in SW948 and SW948-TR. Receptor expression was detected as mean fluorescence intensity (MFI). Values are mean \pm SD of at least three independent experiments. (D) Western blot analysis showing basic expression levels of several proteins involved in apoptosis for SW948 and SW948-TR. One representative of at least three different experiments is shown. Actin is shown as loading control.

\section{Results}

Characteristics of the rhTRAIL-resistant cell line. The TRAILresistant sub-line (SW948-TR) was established from the parental colon carcinoma cell line SW948 following incubation with increasing concentrations of rhTRAIL for 3 months.
The differences in rhTRAIL sensitivity within this isogenic cell model are shown in Fig. 1A. SW948 is extremely sensitive to rhTRAIL with an $\mathrm{IC}_{50}$ of $0.007 \mu \mathrm{g} / \mathrm{ml}$ whereas SW948-TR is resistant to high concentrations of rhTRAIL, or 140-fold less sensitive than its parental cell line. SW948 wells were also more sensitive to rhTRAIL-induced apoptosis than 
A

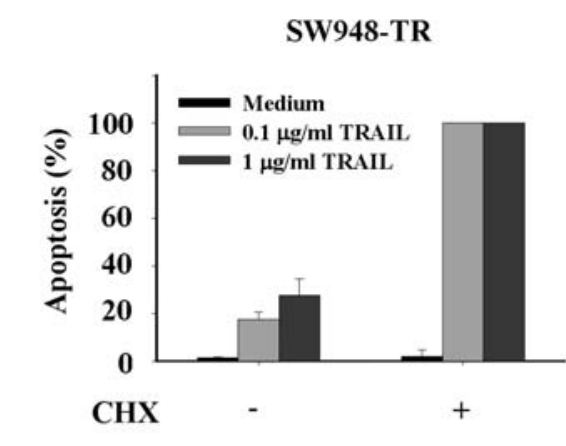

C

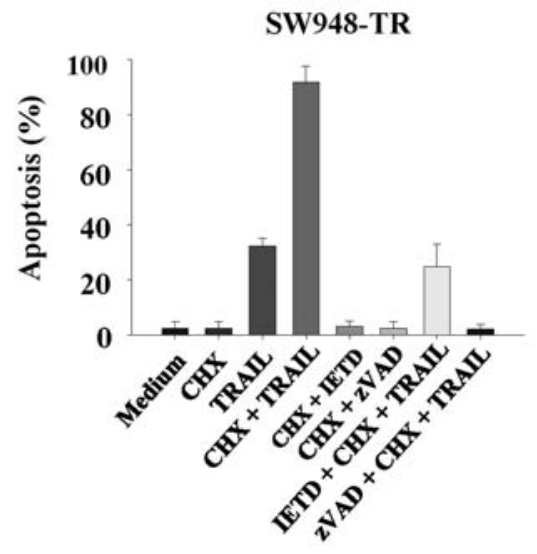

B SW948 SW948-TR $\begin{array}{lllllll}\mathbf{T} & \mathbf{T} & \mathbf{T} & \mathbf{T} & \mathbf{T} & \mathbf{S} & \mathbf{A}\end{array}$

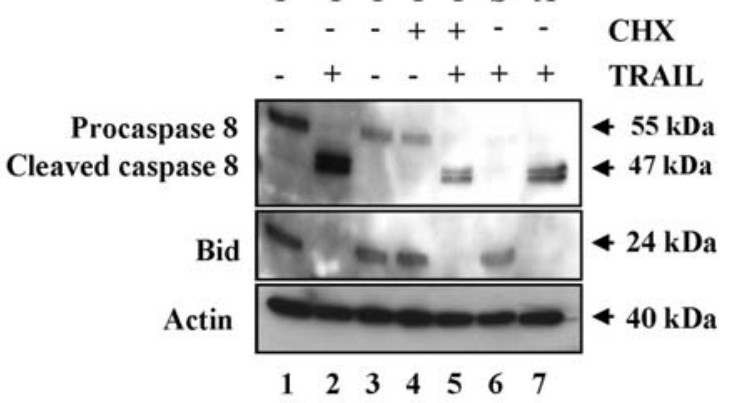

D

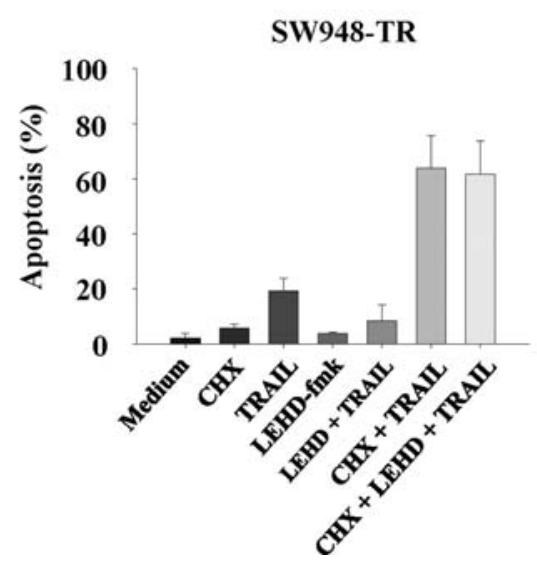

Figure 2. The protein synthesis inhibitor cycloheximide (CHX) sensitizes SW948-TR cells to rhTRAIL-induced apoptosis in a caspase 8-dependent fashion. (A) Sensitization of SW948-TR cells to rhTRAIL-induced apoptosis by CHX, as determined by AO staining. Cells were exposed to 0.1 or $1.0 \mu \mathrm{g} / \mathrm{ml}$ rhTRAIL for $24 \mathrm{~h}$ with or without pre-incubation with $5 \mu \mathrm{g} / \mathrm{ml} \mathrm{CHX}$ for $1 \mathrm{~h}$. Values are mean $\pm \mathrm{SD}$ of at least three independent experiments. (B) Western blot analysis of caspase 8 activation and Bid cleavage in SW948 and SW948-TR cells. Cells were treated with $5 \mu \mathrm{g} / \mathrm{ml} \mathrm{CHX}, 0.1 \mu \mathrm{g} / \mathrm{ml} \mathrm{rhTRAIL}$ alone or in combination. Total cell lysates were loaded (T) and for the SW948-TR cell line, the surviving sub-fraction (S) was separated from the apoptotic (A) cell fraction. Procaspase $8(55 \mathrm{kDa})$ is cleaved in an intermediate product (cleaved caspase 8$)(47 \mathrm{kDa})$ and an active p18 product (18 kDa). Bid (24 kDa) is cleaved in tBid (not detectable) by active caspase 8. Bands of interest are indicated with arrows. (C) Caspase 8-dependent apoptosis in CHX-treated SW948-TR cells. Cells were pre-incubated for $1 \mathrm{~h}$ with $5 \mu \mathrm{g} / \mathrm{ml}$ cycloheximide and/or $50 \mu \mathrm{M}$ zIETD-fmk or $50 \mu \mathrm{M}$ zVAD-fmk before treatment with $0.1 \mu \mathrm{g} / \mathrm{ml}$ rhTRAIL for $4 \mathrm{~h}$. (D) SW948-TR cells were pre-incubated for $1 \mathrm{~h}$ with $5 \mu \mathrm{g} / \mathrm{ml} \mathrm{CHX}$ and/or $50 \mu \mathrm{M}$ zLEHD-fmk before 4 -h-treatment with $0.1 \mu \mathrm{g} / \mathrm{ml}$ rhTRAIL and AO staining for apoptosis.

SW948-TR cells as determined in an apoptosis assay (Fig. 1B). The $\sim 30 \%$ surviving fraction is consistent with previous model of TRAIL resistance in cancer cell lines treated with TRAIL for several months $(17,18)$.

TRAIL receptor membrane expression was evaluated in both cell lines to examine whether TRAIL receptors played a role in the acquisition of TRAIL resistance (Fig. 1C). The cell lines showed similar DR4 $(\mathrm{p}=0.998)$ and DR5 $(\mathrm{p}=0.109)$ membrane expression. DcR1 and DcR2 were not, or hardly expressed on the cell surface of both cell lines. Western blotting was performed to determine whether the acquisition of TRAIL resistance in SW948-TR cells was caused by a change in basal protein expression levels of DISC components, Bcl-2 family members or members of the inhibitor of apoptosis protein (IAP) family (Fig. 1D). Total DR4 and DR5 levels were similar between the two cell lines. No differences in FADD, Bid, Bax, Bak, Bcl-2, Bcl- $\mathrm{X}_{\mathrm{L}}$, or caspase 9 were observed between SW948 and SW948-TR. Members of the IAP family, namely survivin, c-IAP1 and c-IAP2 were similarly expressed in both cell lines. Procaspase 8 levels were, however, lower and procaspase 10 levels slightly higher in SW948-TR than in SW948.

The protein synthesis inhibitor cycloheximide sensitizes SW948-TR cells to rhTRAIL in a caspase 8-dependent manner. In SW948-TR, incubation with rhTRAIL induced apoptosis in $\sim 30 \%$ of cells. The protein synthesis inhibitor cycloheximide strongly sensitized SW948-TR cells to rhTRAIL (Fig. 2A). The levels of apoptosis reached in presence of cycloheximide were similar to the levels of apoptosis initially found in the parental cell line (compare with Fig. 1B, top panel). These results demonstrate that the TRAIL receptor-mediated apoptotic pathway is still functional in SW948-TR cells.

Caspase 8 activation is a crucial step in death receptormediated apoptosis. To study whether inhibition of TRAILinduced apoptosis in SW948-TR originates upstream, downstream, or at the level of caspase 8 , the cleavage of caspase 8 and Bid in response to rhTRAIL was studied by Western blotting. Caspase 8 activation was represented by either the intermediate caspase 8 product ( $\mathrm{p} 45 / 47$ ) or the active caspase 8 
A

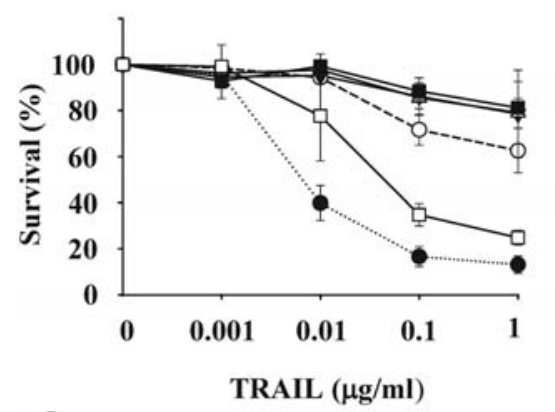

C

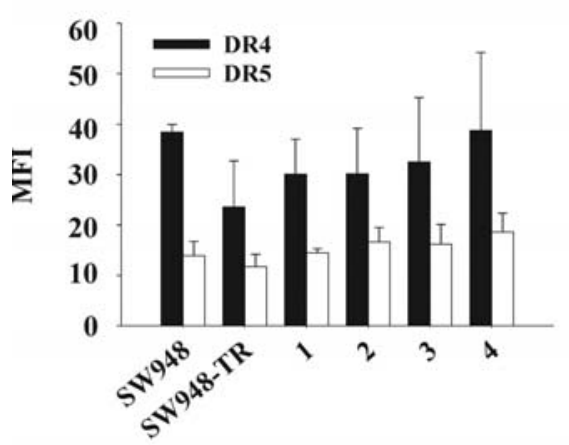

$\mathbf{E}$

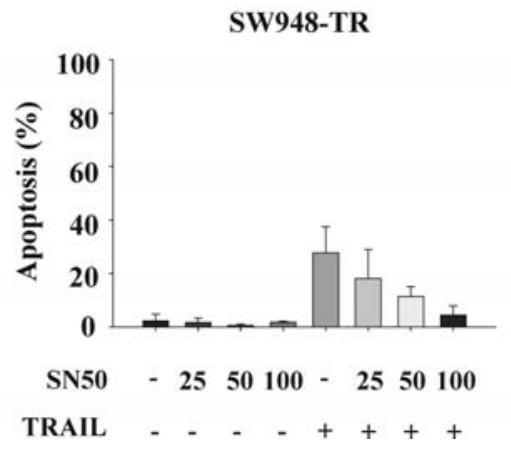

B
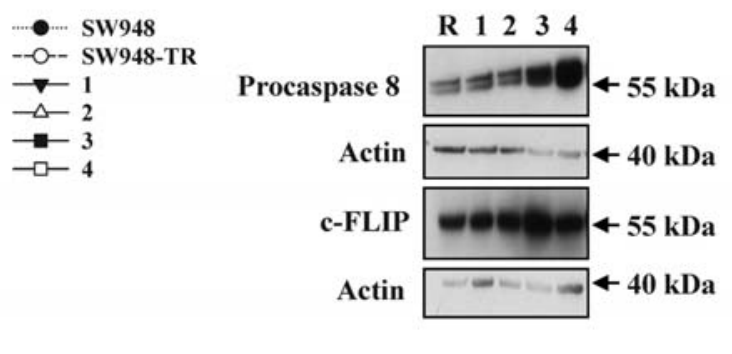

D

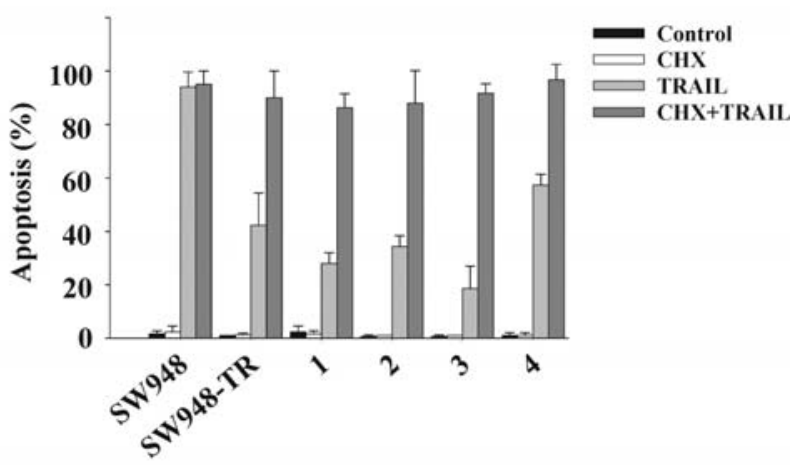

Figure 3. Sub-clones of SW948-TR have similar TRAIL sensitivity. (A) Survival of SW948, SW948-TR and the SW948-TR sub-clones (1, 2, 3 and 4) after continuous incubation with rhTRAIL as measured with cytotoxicity assays. (B) Western blot analysis of basic procaspase 8 and c-FLIP protein expression in SW948-TR (R) and in the SW948-TR sub-clones (1, 2, 3 and 4). Bands of interest are indicated with arrows. (C) Basic membrane expression of the TRAIL receptors DR4 (black bars), DR5 (white bars) in SW948, SW948-TR and in the sub-clones of SW948-TR (clones 1, 2, 3 and 4). Expression was detected as mean fluorescence intensity (MFI) of the whole cell population. (D) Apoptosis induced in SW948, SW948-TR and in the sub-clones of SW948-TR (clones 1, 2,3 and 4). Cells were plated as control (black bars), exposed to $5 \mu \mathrm{g} / \mathrm{ml} \mathrm{CHX}$ (white bars), $0.1 \mu \mathrm{g} / \mathrm{ml} \mathrm{rhTRAIL}$ (light grey bars) or pretreated with $5 \mu \mathrm{g} / \mathrm{ml}$ cycloheximide for $1 \mathrm{~h}$ followed by 5 -h incubation with $0.1 \mu \mathrm{g} / \mathrm{ml}$ rhTRAIL (dark grey bars). (E) The NFkB inhibitor SN-50 does not sensitize SW948-TR to rhTRAIL-induced apoptosis. Cells were pre-exposed to 25,50 or $100 \mu \mathrm{g} / \mathrm{ml} \mathrm{SN-50}$ alone or in combination with $0.1 \mu \mathrm{g} / \mathrm{ml} \mathrm{rhTRAIL}$ for $5 \mathrm{~h}$.

(p18) sub-units, and activation of Bid by disappearance of the full length protein. Exposure of SW948 to rhTRAIL for $5 \mathrm{~h}$ induced cleavage of procaspase 8 as well as Bid activation (Fig. 2B, lane 2). In the presence of cycloheximide and rhTRAIL, caspase 8 cleavage and Bid activation were also observed in SW948-TR (Fig. 2B, lane 5). Since the TRAILsensitive fraction of $\sim 30 \%$ in SW948-TR may have complicated our observations (Fig. 2A), the apoptotic (A) and the surviving (S) fraction of SW948-TR were separated after exposure to rhTRAIL for $5 \mathrm{~h}$. High levels of cleaved caspase 8 and complete Bid activation were detected in the apoptotic fraction (Fig. 2B, lane 7). In contrast, Bid was not cleaved in the surviving fraction where pro- and active caspase 8 were almost undetectable (Fig. 2B, lanes 6). These results strongly suggest that resistance is initiated at the level of caspase 8 in SW948-TR, through downregulation of proand/or active caspase 8 levels.

In presence of cycloheximide, both the caspase 8-specific inhibitor zIETD-fmk and the pan-caspase inhibitor zVADfmk inhibited TRAIL-induced apoptosis in the resistant cell line (Fig. 2C), indicating the importance of caspases in TRAIL-induced apoptosis following cycloheximide treatment. In contrast, the caspase 9 inhibitor zLEHD-fmk could not inhibit TRAIL-induced apoptosis in SW948-TR (Fig. 2D). Thus, in presence of cycloheximide, enough active caspases can be generated without the need for amplification via caspase 9 downstream of the mitochondria (19). 
A

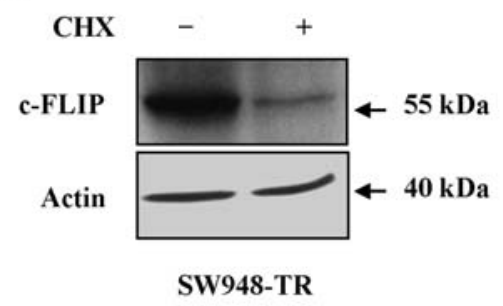

C

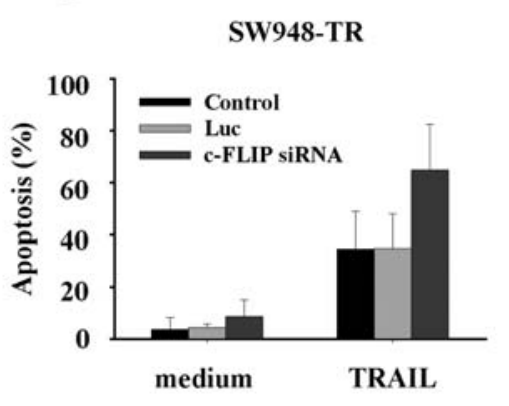

E

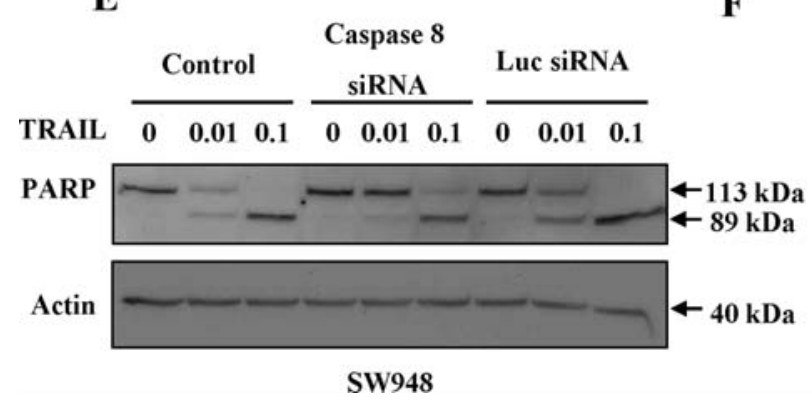

\section{F}

B

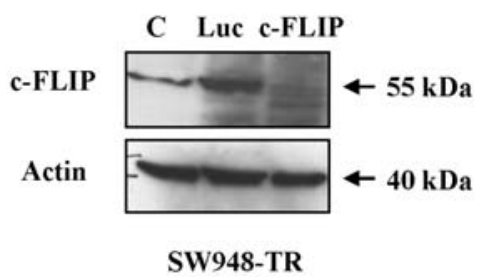

D

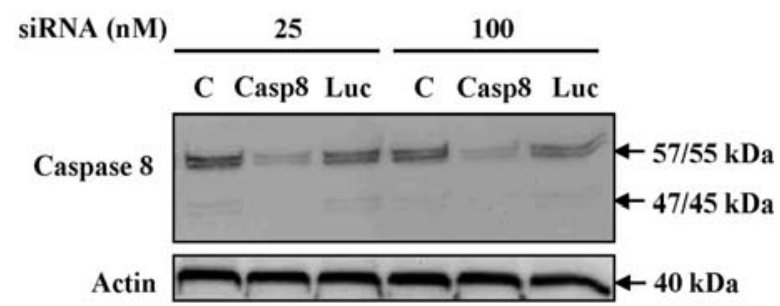

SW948

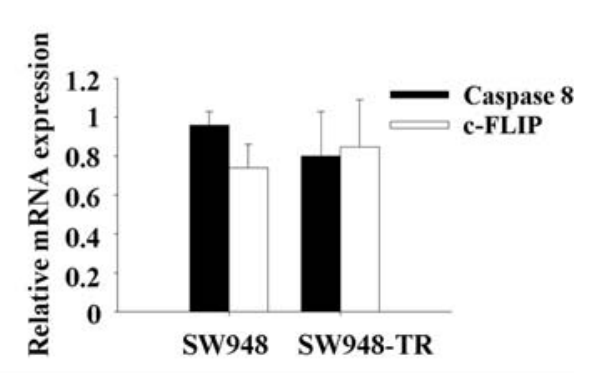

Figure 4. Caspase 8- and FLIP-levels are important regulator of TRAIL sensitivity in the SW948/SW948-TR cell lines. (A) Western blot analysis of c-FLIP protein levels in SW948-TR cells after 24-h incubation with $5 \mu \mathrm{g} / \mathrm{ml} \mathrm{CHX.} \mathrm{(B)} \mathrm{SW948-TR} \mathrm{cells} \mathrm{were} \mathrm{transfected} \mathrm{with} \mathrm{siRNA} \mathrm{duplexes} \mathrm{directed} \mathrm{against} \mathrm{the}$ luciferase gene (Luc) as control siRNA or the c-FLIP gene. Western blot analysis showed downregulation of c-FLIP by c-FLIP siRNAs. (C) Apoptosis assay in siRNA transfected SW948-TR cells. Control cells and luciferase or c-FLIP siRNA transfected cells were plated and 48 h after transfection cells were incubated with rhTRAIL. After 4-5 h rhTRAIL incubation the percentage of apoptosis was determined (p<0.05). (D) Downregulation of caspase 8 by specific siRNAs in SW948-TR. Cells were transfected with 25-100 nM siRNA duplexes targeting luciferase or the caspase 8 mRNA (Casp8) for 48 h before lysis and Western blot analysis. (E) Effects of caspase 8 downregulation on PARP cleavage as determined by Western blot analysis. Cells were transfected with 66 nM siRNA for $48 \mathrm{~h}$ before treatment with rhTRAIL for $5 \mathrm{~h}$ before lysis. (F) Relative caspase 8 and FLIP mRNA levels in SW948 and SW948-TR, as determined by real-time PCR.

Similar phenotypes in sub-clones of SW948-TR. Since cell cycle distribution in the surviving fraction was similar to that of untreated cells, we excluded the possibility that the apoptotic fraction consisted of cells in a specific phase of the cell cycle (data not shown). To test whether SW948-TR consisted of a heterogeneous cell population causing differential expression of caspase 8 in $30 \%$ of the cells, 15 sub-clones were isolated using minimal dilution and soft agar sub-cloning methods. Effects of rhTRAIL incubation on cell survival in SW948, SW948-TR and four randomly-chosen clones are shown in Fig. 3A. Screening for DISC protein expression, we found no difference in membrane TRAIL receptors, procaspase 8 and c-FLIP protein expression levels between the sub-lines (Fig. 3B and C). Remarkably, most sub-clones showed apoptosis in a range of $20-40 \%$ after incubation with rhTRAIL alone, and were completely sensitized to rhTRAIL by cycloheximide (CHX, Fig. 3D). Clone 4 exhibited slightly higher
rhTRAIL sensitivity in survival and apoptosis assay (Fig. 3A and D). This may be explained by the fact that clone 4 exhibited higher caspase 8 expression and similar c-FLIP expression compared to other clones (for example, compare clones 1 and 4 in Fig. 3B). The fact that we could not isolate individual stable TRAIL-sensitive and TRAIL-resistant clones suggest that the $\sim 30 \%$ apoptosis seen in SW948-TR cells after rhTRAIL treatment is a general property of the cell population. The existence of an apoptotic fraction might partly be explained by small cell-to-cell variations in caspase 8 and FLIP levels in favor of apoptosis.

The NFkB pathway has sometimes been attributed proand anti-apoptotic properties as regard to TRAIL-sensitivity $(20,21)$. SW948-TR cells were treated with SN-50, a cell permeable inhibitory peptide based on NFkB p50, to investigate the role of $\mathrm{NF}$ B-activation in response to rhTRAIL (Fig. 3E). Inhibition of NFkB did not sensitize SW948-TR to 
rhTRAIL. Remarkably, SN-50 showed an apoptosis-inhibiting effect on the $\sim 30 \%$ apoptotic cells detected after rhTRAIL exposure, suggesting potential pro-apoptotic effects of the NFKB pathway in the rhTRAIL-sensitive fraction of the SW948-TR cell population.

The caspase 8/c-FLIP ratio plays a role in the differential rhTRAIL sensitivity of SW948 and SW948-TR cells. We previously reported that treating colon carcinoma cell lines with CHX strongly downregulated c-FLIP expression, notably in SW948 cells (15). CHX increased rhTRAIL-sensitivity (Fig. 2A) and downregulated c-FLIP in SW948-TR cells (Fig. 4A). The increase in Bid activation (Fig. 2B) suggests that $\mathrm{CHX}$ primarily modulate rhTRAIL-induced apoptosis upstream of Bid by downregulating c-FLIP and thus facilitating caspase 8 cleavage. However, it cannot be ruled out that CHX inhibits expression of some additional anti-apoptotic proteins with a high turnover. To study the importance of c-FLIP downregulation by CHX in our TRAIL-resistant cell line, we downregulated c-FLIP levels in SW948-TR cells using short interference RNA technology. c-FLIP siRNA specifically downregulated c-FLIP levels compared to luciferase siRNA (Fig. 4A). Downregulating c-FLIP expression sensitized SW948-TR cells to rhTRAIL-induced apoptosis (Fig. 4C). This demonstrates the importance of CHX-induced c-FLIP downregulation for the increase in rhTRAILsensitivity in SW948-TR. Since SW948-TR exhibited lower levels of procaspase 8 rather than having higher levels of its inhibitor c-FLIP (Fig. 1D), we tested the role played by caspase 8 levels in TRAIL sensitivity of the parental cell line. Caspase 8 levels were therefore downregulated using siRNA before TRAIL treatment in the parental cell line (Fig. 4D). Cleavage of the executioner caspase substrate PARP was used to evaluate rhTRAIL-induced apoptosis in SW948 cells following caspase 8 downregulation. As seen in Fig. 4E, treatment with $0.01 \mu \mathrm{g} / \mathrm{ml}$ rhTRAIL induced cleavage of $\sim 50 \%$ of the available full-length PARP into its active form $(89 \mathrm{kDa})$, both in control and in luciferase-transfected cells. At the same rhTRAIL concentration, caspase 8 downregulation almost completely prevented PARP cleavage in SW948. Thus, decreasing basal caspase 8 expression levels is sufficient to inhibit rhTRAIL-induced apoptosis in SW948 cells, despite the presence of caspase 10 (Fig. 1D). We did not completely prevent rhTRAIL-induced PARP cleavage in SW948 by inhibiting caspase 8 expression, in particular at higher concentrations of rhTRAIL (Fig. 4E). This could be due to incomplete downregulation of caspase 8 (Fig. 4D). Although procaspase 8 protein levels are lower in SW948-TR than in SW948 (Fig. 1D), we now show that mRNA levels of caspase 8 (and c-FLIP) are similar between the two cell lines (Fig. 4F), indicating that the decrease in caspase 8 levels in SW948-TR is due to decreased translation and/or decreased stability of the protein. Altogether, these results indicate that the caspase $8 / \mathrm{c}$-FLIP ratio plays an important role for rhTRAIL sensitivity in SW948 and SW948-TR cells.

MG132 stabilized active caspase 8 protein levels and sensitized SW948-TR to rhTRAIL. As an alternative to c-FLIP downregulation using $\mathrm{CHX}$, restoring caspase 8 levels may also restore rhTRAIL sensitivity in SW948-TR cells. To elucidate

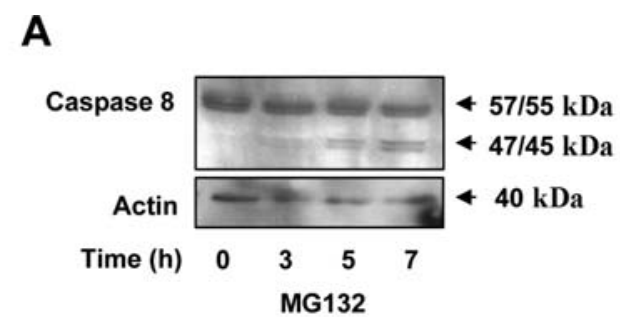

B

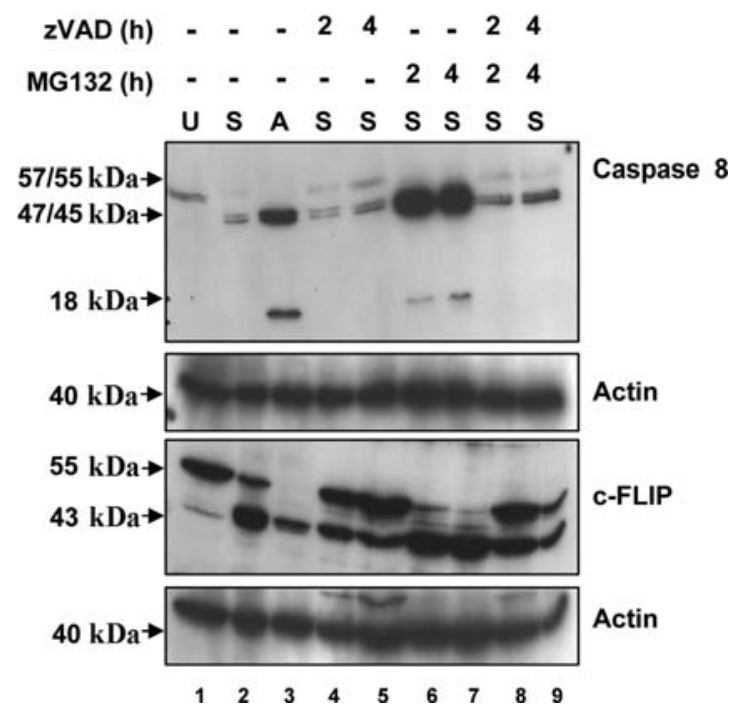

Figure 5. MG132 stabilizes active caspase 8 protein levels and sensitizes SW948-TR to TRAIL in a caspase-dependent manner. (A) Western blot analysis of caspase 8 and c-FLIP protein levels in SW948-TR cells incubated with MG132 for 0-7 h. (B) Western blot analysis of caspase 8 activation and c-FLIP processing in SW948-TR. After 5 h-rhTRAIL incubation $(0.1 \mu \mathrm{g} / \mathrm{ml})$ the surviving cell fraction (S) was separated from the apoptotic cell fraction (A). $\mathrm{U}$ is untreated total cell population. Cells were then exposed to $\mathrm{zVAD}$ and or MG132 for 2 or $4 \mathrm{~h}$

whether rhTRAIL resistance could be caused by increased proteasomal degradation of procaspase 8 levels, SW948-TR cells were treated with the proteasome inhibitor MG132 (Fig. 5A). MG132 treatment did not increase procaspase 8 levels but induced a small increase in the $45 / 47 \mathrm{kDa}$ intermediate product of caspase 8 . No effect on full length c-FLIP levels was found.

The importance of caspase 8 expression levels was studied in the rhTRAIL-treated surviving fraction of SW948-TR (Fig. 5B, lane 2) which expressed low caspase 8 levels. Following rhTRAIL incubation for $5 \mathrm{~h}$, apoptotic cells were washed away and the surviving fraction was incubated with MG132 for an additional 2-4 h (Fig. 5B, lanes 6 and 7). This induced high levels of both the intermediate product and the active form of caspase 8 . The effect of MG132 treatment on c-FLIP levels was also assessed to provide an additional read-out for DISC activity (Fig. 5B). In the surviving fraction, although some cleavage of c-FLIP was detected in presence of rhTRAIL, full-length c-FLIP was still available (Fig. 5B, compare lanes 2 to 3 ). While exposure to MG132 strongly increased intermediate and active caspase 8 levels in the surviving fraction, it only induced a slight augmentation in c-FLIP cleavage (Fig. 5B, compare lanes 6 and 7 with lane 2). 
MG132 neither had effect on DR4 or DR5 protein levels nor on surface expression of these two receptors (data not shown). This indicates that the high levels of active caspase 8 found after MG132 treatment are not caused by an increase in death receptor or procaspase 8 levels. Altogether, these experiments support a role for MG132 in stabilizing active caspase 8 generated at the DISC by TRAIL treatment.

\section{Discussion}

Previous research on long-term acquired TRAIL resistance mechanisms in colon carcinoma cell line reported the accumulation of Bax mutations in vitro and in vivo (9). Because most colorectal cancers are MMR-proficient, we analyzed the mechanisms of acquired rhTRAIL resistance in an MMR-proficient colon carcinoma cell line model. We now show that in these cells, resistance to rhTRAIL is caused by increased degradation of the pool of activated caspase 8 . These results point toward preservation of the early TRAIL resistance mechanism and ressembles results obtained in MMR-deficient TRAIL-resistant colon cancer cells obtained following four rounds of infection with TRAIL adenoviral vectors (22). In support of a generally critical involvement of active caspase destabilization mechanisms in TRAIL resistance, the stability of rhTRAIL-induced active caspase 3 (but not caspase 8) was found to be lower in an MMRproficient ovarian cancer cell line exposed to rhTRAIL for 20 weeks.

The TRAIL-resistant SW948-TR cell line was established from the extremely TRAIL-sensitive SW948 colon carcinoma cell line after three months of continuous incubation with rhTRAIL. TRAIL receptor membrane expression was similar between the two cell lines. CHX sensitized SW948-TR cells to rhTRAIL as indicated by increased activation of both caspase 8 and Bid. Although SW948 and SW948-TR cells had similar c-FLIP protein levels, procaspase 8 levels were lower in SW948-TR, implying reduction of the caspase 8/cFLIP ratio. We show that this ratio plays a crucial role in regulating TRAIL sensitivity in these cells. Downregulating c-FLIP protein levels with CHX increased rhTRAIL-sensitivity in SW948-TR cells. Treatment of SW948-TR with specific c-FLIP siRNA also sensitized SW948-TR cells to rhTRAIL, while downregulation of caspase 8 decreased rhTRAIL sensitivity in SW948. In rhTRAIL-treated SW948-TR cells, separation of the rhTRAIL-resistant surviving cell fraction from the apoptotic fraction showed that procaspase 8 levels were lower in the surviving fraction than in the apoptotic fraction or in the sensitive parental cell line. Additionally, only partial cleavage of full-length c-FLIP was found in the surviving cell fraction of SW948-TR, suggesting that the decrease in rhTRAIL-induced apoptosis was associated with lower DISC activity. Spencer et al recently demonstrated that the so-called 'fractional killing' of TRAIL was caused by cell-to-cell variability in protein expression due to noise in gene expression (23). The presence of a CD133 positive sub-population could also explain this differential killing of rhTRAIL $(24,25)$. Altogether, our results support a key role for the caspase 8/c-FLIP ratio in initiating the acquired rhTRAIL resistance of SW948-TR cells. Accordingly, several studies have shown that a low caspase 8/c-FLIP ratio contributes to poor TRAIL sensitivity in cancer cell lines (26-31). Previously, we have reported that both c-FLIP and caspase 8 expressions are increased in colon cancer compared to normal epithelium (32). These changes were accompanied by large variations in caspase $8 / \mathrm{c}$-FLIP ratio between colon tumors, which suggest the presence of subgroups of patients with pro- and anti-apoptotic caspase 8/c-FLIP ratios.

We further investigated the decrease in caspase 8 in the TRAIL-resistant cell lines. Changes cell cycle distribution could not explain the differences in caspase 8 between SW948 and SW948-TR. Several studies observed downregulation of caspase 8 via hypermethylation of the caspase 8 promoter $(33,34)$. Treatment of SW948-TR with the demethylating agent 5-aza-2 deoxycytidine had no effect on caspase 8 levels (data not shown). This finding is in line with the comparable mRNA levels present in SW948 and SW948-TR, indicating either decreased translation and/or decreased stability of caspase 8 in SW948-TR. As previously observed by Zhang et $\mathrm{al}$, proteasome inhibition could not restore procaspase 8 levels (22). SW948-TR cells could, however, be sensitized to rhTRAIL by MG132, in association with an increase in intermediate and active caspase 8 levels. These results suggest that proteasome inhibition prevented the degradation of the cleaved forms of caspase 8 .

Little is known about the degradation mechanism(s) of procaspase 8 or active caspase 8 . Bi-functional apoptosis regulator protein (BAR) was found to bind both forms of the protein. BAR possesses a RING domain with putative ubiquitin-protein isopeptide ligase-like activity. Stegh et al speculated that active caspase 8 might be targeted for ubiquitination and proteasome-dependent degradation, although proteasome inhibitors failed to prevent caspase 8 degradation (35). Previously, a family of apoptotic inhibitors, the caspases 8 and 10 associated RING proteins (CARPs) has been identified (36). Binding of CARP1 and CARP2 negatively regulated death effector domain (DED) caspases by contributing to their ubiquitin-mediated proteolysis. Knockdown of CARPs did not result in an up-regulation of procaspase 8 but both TRAIL- and Fas-induced apoptosis were increased via enhanced caspase 8 processing. In Drosophila, MG132 treatment resulted in an accumulation of the processed form of the apical caspase DRONC but not of its full-length form (37). Recently, Jin et al showed that consecutively to TRAIL treatment, a cullin3 (CUL3)-based E3 ligase induces polyubiquination of caspase 8 at the DISC, thereby causing caspase 8 aggregation and full activation of this protein (38). The authors also found that proteasome inhibition did not stabilize poly-ubiquitinated active caspase 8 in the moderately TRAIL-sensitive H460 cell line. In Bid-depleted HeLa cells treated with $\mathrm{CHX}$, active caspase 8 activity persisted for several hours after TRAIL treatment (39). Altogether, these results suggest that proteasomal degradation might be specific for the activated forms of caspase 8 , and more prevalent in rhTRAIL-resistant cells.

A limited number of studies have looked at mechanisms of acquired TRAIL resistance by long-term culturing of cells in medium containing rhTRAIL. TRAIL resistance was associated with a change in TRAIL receptor membrane expression in osteosarcomas (40) and in melanomas (41). 
In another study TRAIL exposure selected for cells with Bax mutations in MMR-deficient colon carcinoma cell lines (9). The importance of caspase 8 in TRAIL sensitivity has been shown before in non-isogenic cell lines $(23,26,27,42)$. Active caspase 8 degradation was involved in the TRAIL resistance of an MMR-deficient colon cancer sub-cell line obtained after four rounds of selection with adenoviral TRAIL vectors (22). We are, however, the first to show that increased degradation of active caspase 8 still plays a crucial role in rhTRAIL-resistant colon carcinoma cells obtained after longterm exposure to this agent.

In conclusion, acquired rhTRAIL-resistance in SW948TR is caused by decreased basal procaspase 8/c-FLIP ratio and increased degradation of cleaved caspase 8 after rhTRAIL treatment. Modulating the procaspase 8/c-FLIP ratio using c-FLIP siRNA or by stabilizing active caspase 8 protein levels using proteasome inhibition can restore rhTRAIL sensitivity in MMR-proficient cells which have acquired rhTRAIL resistance. These results may help overcoming TRAIL resistance using rational strategies with targeted drugs.

\section{Acknowledgements}

This work was supported by grant RUG 2000-2286 and grant RUG 2005-3361 from the Dutch Cancer Society and a grant from the Maurits en Anna de Kock Stichting.

\section{References}

1. Wiley SR, Schooley K, Smolak PJ, et al: Identification and characterization of a new member of the TNF family that induces apoptosis. Immunity 3: 673-682, 1995

2. Pitti RM, Marsters SA, Ruppert S, Donahue CJ, Moore A and Ashkenazi A: Induction of apoptosis by Apo-2 ligand, a new member of the tumor necrosis factor cytokine family. J Biol Chem 271: 12687-12690, 1996.

3. Koschny R, Walczak H and Ganten TM: The promise of TRAIL-potential and risks of a novel anticancer therapy. J Mol Med 85: 923-935, 2007.

4. Mahalingam D, Szegezdi E, Keane M, De Jong S and Samali A: TRAIL receptor signalling and modulation: are we on the right TRAIL? Cancer Treatment Rev 35: 280-288, 2009.

5. Oldenhuis CNAM, Stegehuis JH, Walenkamp AME, De Jong S and De Vries EGE: Targeting TRAIL death receptors. Curr Opin Pharmacol 8: 433-439, 2008

6. Duiker EW, Mom CH, De Jong S, Willemse PH, Gietema JA, van der Zee AG and De Vries EGE: The clinical trail of TRAIL. Eur J Cancer 42: 2233-2240, 2006.

7. Ashkenazi A, Pai RC, Fong S, et al: Safety and antitumor activity of recombinant soluble Apo2 ligand. J Clin Invest 104: 155-162, 1999.

8. Falschlehner $\mathrm{C}$, Emmerich $\mathrm{CH}$, Gerlach B and Walczak $\mathrm{H}$ : TRAIL signalling: decisions between life and death. Int $\mathrm{J}$ Biochem Cell Biol 39: 1462-1475, 2007.

9. Le Blanc H, Lawrence D, Varfolomeev E, et al: Tumor-cell resistance to death receptor-induced apoptosis through mutational inactivation of the proapoptotic Bcl-2 homolog Bax. Nat Med 8: 274-281, 2002

10. Ionov Y, Peinado MA, Malkhosyan S, Shibata D and Perucho M: Ubiquitous somatic mutations in simple repeated sequences reveal a new mechanism for colonic carcinogenesis. Nature 363 . 558-561, 1993.

11. Rampino N, Yamamoto H, Ionov Y, Li Y, Sawai H, Reed JC and Perucho M: Somatic frameshift mutations in the BAX gene in colon cancers of the microsatellite mutator phenotype. Science 275: 967-969, 1997.

12. Peltomaki P: Deficient DNA mismatch repair: a common etiologic factor for colon cancer. Hum Mol Genet 10: 735-740, 2001 .
13. Burns TF and El Deiry WS: Identification of inhibitors of TRAIL-induced death (ITIDs) in the TRAIL-sensitive colon carcinoma cell line SW480 using a genetic approach. J Biol Chem 276: 37879-37886, 2001.

14. Melcher R, Koehler S, Steinlein C, et al: Spectral karyotype analysis of colon cancer cell lines of the tumor suppressor and mutator pathway. Cytogenet Genome Res 98: 22-28, 2002.

15. Van Geelen CMM, De Vries EGE, Le TKP, van Weeghel RP and De Jong S: Differential modulation of the TRAIL receptors and the CD95 receptor in colon carcinoma cell lines. Br J Cancer 89: 363-373, 2003.

16. Elbashir SM, Harborth J, Lendeckel W, Yalcin A, Weber K and Tuschl T: Duplexes of 21-nucleotide RNAs mediate RNA interference in cultured mammalian cells. Nature 411: 494-498, 2001.

17. Lane D, Côté M, Grondin R, Couture M and Piché A: Acquired resistance to TRAIL-induced apoptosis in human ovarian cancer cells is conferred by increased turnover of mature caspase- 3 . Mol Cancer Ther 5: 509-521, 2006.

18. Lee TJ, Lee JT, Park JW and Kwon TK: Acquired TRAIL resistance in human breast cancer cells are caused by the sustained cFLIP(L) and XIAP protein levels and ERK activation. Biochem Biophys Res Commun 351: 1024-1030, 2006.

19. Barnhart BC, Alappat EC and Peter ME: The CD95 type I/type II model. Semin Immunol 15: 185-193, 2003.

20. Plantivaux A, Szegezdi E, Samali A and Egan L: Is there a role for nuclear factor kappaB in tumor necrosis factor-related apoptosis-inducing ligand resistance? Ann NY Acad Sci 1171: 38-49, 2009.

21. Radhakrishnan SK and Kamalakaran S: Pro-apoptotic role of NF-kappaB: implications for cancer therapy. Biochim Biophys Acta 1766: 53-62, 2006

22. Zhang L, Zhu H, Teraishi F, Davis JJ, Guo W, Fan Z and Fang B: Accelerated degradation of caspase- 8 protein correlates with TRAIL resistance in a DLD1 human colon cancer cell line. Neoplasia 7: 594-602, 2005.

23. Spencer SL, Gaudet S, Albeck JG, Burke JM and Sorger PK: Non-genetic origins of cell-to-cell variability in TRAIL-induced apoptosis. Nature 459: 428-432, 2009.

24. Sussman RT, Ricci MS, Hart LS, Sun SY and El Deiry WS: Chemotherapy-resistant side-population of colon cancer cells has a higher sensitivity to TRAIL than the non-SP, a higher expression of c-Myc and TRAIL-receptor DR4. Cancer Biol Ther 6: 1490-1495, 2007.

25. Zobalova R, Stantic M, Prokopova K, Dong LF and Neuzil J: Cancer cells with high expression of CD133 exert FLIP upregulation and resistance to TRAIL-induced apoptosis. Biofactors 34: 231-235, 2009

26. Harper N, Farrow SN, Kaptein A, Cohen GM and MacFarlane M: Modulation of tumor necrosis factor apoptosis-inducing ligandinduced NF-kappa B activation by inhibition of apical caspases. J Biol Chem 276: 34743-34752, 2001.

27. Lippa MS, Strockbine LD, Le TT, Branstetter DG, Strathdee CA and Holland PM: Expression of anti-apoptotic factors modulates Apo2L/TRAIL resistance in colon carcinoma cells. Apoptosis 12: 1465-1478, 2007.

28. MacFarlane M, Harper N, Snowden RT, Dyer MJ, Barnett GA, Pringle JH and Cohen GM: Mechanisms of resistance to TRAIL-induced apoptosis in primary B cell chronic lymphocytic leukaemia. Oncogene 21: 6809-6818, 2002.

29. MacFarlane M: TRAIL-induced signalling and apoptosis. Toxicol Lett 139: 89-97, 2003.

30. Ganten TM, Haas TL, Sykora J, et al: Enhanced caspase-8 recruitment to and activation at the DISC is critical for sensitisation of human hepatocellular carcinoma cells to TRAILinduced apoptosis by chemotherapeutic drugs. Cell Death Differ 11 (Suppl. 1): S86-S96, 2004.

31. Pennarun B, Meijer A, De Vries EGE, Kleibeuker JH, Kruyt FA and De Jong S: Playing the DISC: turning on TRAIL death receptor-mediated apoptosis in cancer. Biochim Biophys Acta (BBA) - Rev Cancer 1805: 123-140, 2010.

32. Heijink DM, Kleibeuker JH, Jalving M, Boersma-van Ek W Koornstra JJ, Wesseling J and De Jong S: Independent induction of caspase- 8 and cFLIP expression during colorectal carcinogenesis in sporadic and HNPCC adenomas and carcinomas. Cell Oncol 29: 409-419, 2007.

33. Fulda S, Kufer MU, Meyer E, van Valen F, DockhornDworniczak B and Debatin KM: Sensitization for death receptoror drug-induced apoptosis by re-expression of caspase- 8 through demethylation or gene transfer. Oncogene 20: 5865-5877, 2001. 
34. Harada K, Toyooka S, Shivapurkar N, et al: Deregulation of caspase 8 and 10 expression in pediatric tumors and cell lines. Cancer Res 62: 5897-5901, 2002.

35. Stegh AH, Barnhart BC, Volkland J, Algeciras-Schimnich A, $\mathrm{Ke} \mathrm{N}$, Reed JC and Peter ME: Inactivation of caspase- 8 on mitochondria of Bcl-xL-expressing MCF7-Fas cells: role for the bifunctional apoptosis regulator protein. J Biol Chem 277: 4351-4360, 2002.

36. McDonald ER, III and El Deiry WS: Suppression of caspase-8and -10-associated RING proteins results in sensitization to death ligands and inhibition of tumor cell growth. Proc Natl Acad Sci USA 101: 6170-6175, 2004.

37. Muro I, Hay BA and Clem RJ: The Drosophila DIAP1 protein is required to prevent accumulation of a continuously generated, processed form of the apical caspase DRONC. J Biol Chem 277: 49644-49650, 2002.

38. Jin Z, Li Y, Pitti R, Lawrence D, Pham VC, Lill JR and Ashkenazi A: Cullin3-based polyubiquitination and p62dependent aggregation of caspase- 8 mediate extrinsic apoptosis signaling. Cell 137: 721-735, 2009.
39. Hellwig C, Kohler B, Lehtivarjo A, Dussmann H, Courtney M, Prehn J and Rehm M: Real-time analysis of tumor necrosis factor-related apoptosis-inducing ligand/cycloheximide-induced caspase activities during apoptosis initiation. J Biol Chem 283: 21676-21685, 2008.

40. Bouralexis S, Findlay DM, Atkins GJ, Labrinidis A, Hay S and Evdokiou A: Progressive resistance of BTK-143 osteosarcoma cells to Apo2L/TRAIL-induced apoptosis is mediated by acquisition of DcR2/TRAIL-R4 expression: resensitisation with chemotherapy. Br J Cancer 89: 206-214, 2003.

41. Nguyen T, Zhang XD and Hersey P: Relative resistance of fresh isolates of melanoma to tumor necrosis factor-related apoptosisinducing ligand (TRAIL)-induced apoptosis. Clin Cancer Res 7: S966-S973, 2001.

42. Teitz T, Lahti JM and Kidd VJ: Aggressive childhood neuroblastomas do not express caspase-8: an important component of programmed cell death. J Mol Med 79: 428-436, 2001. 